\title{
Fisiología y ecología del fitocromo: su función en las semillas.
}

\author{
Alma OroZCO-SEGOVIA ${ }^{1}$
}

\begin{abstract}
RESUMEN. En los procesos regulados por la luz el fitocromo es el pigmento fotorreceptor. En los últimos años se le ha conferido a este pigmento un papel importante en la detección de las condiciones ambientales de los procesos regulados por él (fotomorfogénesis, fotoblastismo). Sin embargo estos procesos no están del todo estudiados desde el punto de vista ecofisiológico, por lo que resulta difícil su adecuada interpretación. En el presente trabajo se analizan algunos de los trabajos realizados sobre el fotoblastismo (germinación regulada por la luz) en semillas de especies pioneras de la selva tropical de la Estación de Biología Tropical "Los Tuxtlas", Veracruz, con el fin de conocer con mayor precisión la función del fitocromo desde el punto de vista ecológico.
\end{abstract}

ABSTRACT.. Phytochrome is the plant pigment which participate in several developmental processes regulated by light. In recent years this pigment has been associated with the detection of the environmental conditions but the re is still a gap of information concerning the physioecological role of the pigment. In this paper the knowledge of the role of phytochrome on seed photoblastism is analysed based on several recent works done on the subject mainly with pioneer rain forest plants from Los Tuxtlas, Veracruz.

\section{ANTECEDENTES}

En todo proceso regulado por la luz se requiere de un pigmento que tenga una función receptora y que a la vez actúe como un desencadenador o inhibidor de los procesos fisiológicos involucrados. En el fotoblastismo y en la fotomorgénesis el pigmento fotorreceptor es el fitocromo, el cual fué descrito por Borthwick et al (1952) en un experimento realizado con semillas de lechuga de la variedad Grand Rapids, en él se detectó que la luz roja $(660 \mathrm{~nm})$ activa el fitocromo y que la luz ultrarroja o roja lejana $(730 \mathrm{~nm})$ lo inactiva.

1 Centro de Ecología, Universidad Nacional Autónoma de México, Apdo. Postal 70-275, Delegación Coyoacan 04510, México, D.F.

Orozco-Segovia A. 1989. Fisiología y ecología del fitocromo: su función en las semillas. Boletín de la Sociedad Botánica de México 49: 71-84. 
A partir de su descubrimiento se desarrollaron un gran número de trabajos principalmente de indole fisiológico que han permitido conocer muchas características del fitocromo, de las cuales sólo nos referiremos a las más estrechamente vinculadas con la germinación fotorregulada o fotoblastismo.

La relación entre el fitocromo activo y el contenido total de fitocromo (Pfr/Pt) de una semilla puede variar debido a: el grado de hidratación del pigmento y a los procesos de nueva síntesis, reversión ( $\mathrm{Pfr}$ a $\mathrm{Pr}$ ) o reversión inversa ( $\mathrm{Pr}$ a Pfr), que sufre el fitocromo durante el proceso de formación de la semilla o durante la vida de ésta (Smith, 1982). A la relación Pfr/Pt, se le conoce como fotoequilibrio y el valor de éste difiere dependiendo de la composición espectral de la luz bajo la cual se encuentre la semilla, por ejemplo: la luz azul produce un fotoequilibrio de 0.35 , la luz roja lejana de 0.05 y la roja de 0.8 . El fotoequilibrio alcanzado con cada tipo de luz no determina el que una especie germine o no, esto depende de que la especie alcance su propio umbral de respuesta, ya que el nivel requerido de Pfr, en relación al Pt, varía en cada especie y depende de otras condiciones ambientales como podrían ser la temperatura y en algunos casos, la tasa de destrucción y síntesis del fitocromo que presenta la especie.

Las semillas, cuya respuesta a la luz se conoce, se han dividido básicamente en tres grupos: fotoblásticas positivas, no germinan en la oscuridad y constituye el $70 \%$ de las especies; fotoblásticas negativas, su germinación es inhibida por la luz, son el $25 \%$ de las especies; por último las especies indiferentes a la luz, constituido únicamente por el 5\% de las especies (Come, 1970). En la formación de estos grupos no han sido consideradas muchas especies tropicales, sobre todo las semillas de los árboles de la selva madura cuya germinación es seguramente indiferente a la luz, además se han encontrado un gran número de variaciones en estos patrones básicos por lo que en la actualidad esta clasificación resulta inoperante; sin embargo, gran parte de la literatura sobre el tema hace referencia a ella.

Tanto en semillas fotoblásticas positivas como en fotoblásticas negativas la luz roja estimula la germinación, mientras que la luz roja lejana la inhibe. En las fotoblásticas negativas hay una diferente reactividad del pigmento fitocromo a ambas calidades de luz, lo que provoca la inhibición de la germinación cuando se les expone simultáneamente a ambas calidades contenidas en la luz blanca (LB) (Evenari, 1965; Satter y Galston, 1977).

El trabajo de Cumming (1963) sobre la inhibición de la germinación de Chenopodium por el dosel vegetal y los estudios realizados sobre la composición espectral de la luz que ocurre en forma natural en las diferentes comunidades vegetales, rica en rojo lejano, han dado lugar al desarrollo de una gran cantidad de investigaciones sobre el efecto de los doseles vegetales en la germinación y su significado ecológico. En forma simultánea los trabajos de Feltner y Vasecky (1968) y Wesson y Wareing (1979 a, b), Karssen (1980/1981 a, b) y un gran número de trabajos desarrollados sobre el banco de semillas de diferentes comunidades han hecho que el fotoblastismo sea un fenómeno claramente asociado con la permanencia de las semillas en el suelo.

Como una consecuencia de estos trabajos se ha llegado a la conclusión de que el fotoblastismo, o sensibilidad a la luz regulada por el fitocromo, es una característica frecuente, en las semillas de especies heliófilas y puede decirse que la función fundamental del fitocromo en las semillas consiste en imponer la latencia cuando las condiciones 
lumínicas son desfavorables para el establecimiento de las plantas, tanto por el efecto de la existencia de un dosel que reduce el valor de la relación rojo/rojo lejano (R:RL), como por el efecto del suelo, cuando las semillas se encuentran enterradas (Fenner, 1985).

Estos hechos han sido puestos en consideración en el estudio de la dinámica de las comunidades naturales, y las selvas tropicales no han sido la excepción ya que un considerable número de especies colonizadoras de claros son sensibles a la luz (Whitmore, 1983; Vázquez-Yanezy Orozco-Segovia, 1984), por lo que en los últimos años han cobrado gran interés los estudios de la sensibilidad a la luz en estas especies y su papel en colonización de claros, a partir de semillas presentes en el suelo (Vázquez-Yanes y Smith, 1982; Vázquez-Yanes y Orozco-Segovia, 1982, 1987).

Sin embargo, la interpretación ecológica del fotoblastismo no es tan simple debido a que las semillas en la naturaleza pasan inicialmente por un proceso de diseminación que puede tener efectos directos, en el caso de las semillas endozoocoras, y/o indirectos como son el quedar expuestas a condiciones de humedad e iluminación diferentes dependiendo del lugar a donde son llevadas, aunado a esto está el hecho de que de no germinar inmediatamente pasan a formar parte del banco de propágulos del suelo, en donde las semillas interactúan con un gran número de factores ambientales como son: temperatura, luz, niveles de $\mathrm{CO} 2, \mathrm{O} 2$, humedad, predadores, parásitos, etc., que tienen un efecto sobre la latencia y/o sobrevivencia de las semillas en el suelo (Karssen 1980/1981a, b; Martínez-Ramos y Alvarez-Buylla 1986).

Ahora bien como un marco para la complejidad de los hechos que acompañan a la semilla desde su separación de la planta madre hasta el momento de su germinación está el hecho de que las condiciones de luz y temperatura en las que se desarrolla la floración y fructificación afectan la respuesta germinativa. También se ha visto que incluso los fotoperiodos previos a la floración influyen en la germinación (Koller, 1962; Gutterman, 1974; Wurzburger y Koller, 1976; Kigel, et al.,1977, 1979). Por otra parte, la presencia de estructuras fotosintéticas en la semilla afecta su germinabilidad, ya que actúan como filtros de luz, dejando pasar mayores proporciones de RL (Cresswell y Grime, 1981). Este factor sumado al grado de madurez de la semilla, la época de la recolección y las condiciones de luz durante el periodo de secado en conjunto, determinan la respuesta germinativa a la luz.

Alexander y Wulff (1985) consideran que estos efectos llegan no sólo a afectar a las semillas en su respuesta a la luz y en el peso de ésta, si no que afectan los primeros estadios de la plántula e incluso a las semillas de la segunda generación.

Los resultados aquí presentados son la recopilación de la información obtenida en diferentes trabajos realizados en México, que pueden ilustrarnos sobre el comportamiento del fitocromo en condiciones naturales y de laboratorio. Las especies presentadas son fotoblásticas, no germinan ni en la oscuridad ni en rojo lejano al momento de la recolecta (directamente de la planta madre) (Vázquez-Yanes, 1976), y son: Cecropia obtusifolia Bertol (Moraceae), Eupatorium galeotti Rob ex Cher (compositae), Myriocarpa longipes (L.) Blume, Piper aequale Vahl., P. auritum H. B. K., P. aff hispidum Sw., $P$. umbellatum L. (Piperaceae), Urera caracasana (Jacq) Griseb. (Urticaceae).

La metodología empleada en los diferentes trabajos es muy similar por lo que los resultados obtenidos son comparables entre sí, los métodos pueden verse con detalle en 
Vázquez-Yanes y Orozco-Segovia (1987) y Orozco-Segovia et al.„1987); además tanto la recolección de las semillas como el trabajo de campo fue realizado, en todos los casos en la Estación de Biología Tropical "Los Tuxtlas", Veracruz, México. Esta reserva biológica está cubierta por selva alta perennifolia (Miranda y Hernández, 1963), se encuentra a una elevación de $160 \mathrm{~m} \mathrm{~s} \mathrm{n} \mathrm{m}$, presenta una precipitación anual de $4900 \mathrm{~mm}$ y su temperatura media es de $27^{\circ} \mathrm{C}$ (Estrada et al. 1985).

\section{ANÁLISIS DE LA INFORMACIÓN}

En semillas fotoblasticas de especies pioneras encontramos sofisticados mecanismos de detección de las condiciones de iluminación en que germinan, el caso más claro es el de $P$. auritum y $P$. umbellatum, estas especies requieren por lo menos de 5 días de exposiciones consecutivas a la luz $(12 \mathrm{~h})$ para iniciar la germinación y de más días para completarla, además en experimentos de breve duración, en los que se expuso diariamente a las semillas a la luz de la selva (rica en rojo lejano) y a la luz directa (rica en rojo), ambas especies requirieron de $8 \mathrm{~h}$ de luz directa, diariamente, para completar su germinación. Esta información nos indica que estas especies pueden detectar la existencia de condiciones adecuadas para su germinación y establecimiento. En otras especies fotoblásticas, como $P$. aff hispidum y $P$. aequale su requerimiento de luz sólo les indicaría su proximidad a la superficie del suelo, estando enterradas, ya que son especies capaces de germinar bajo un dosel vegetal (Fig. 1). Es importante señalar que los resultados obtenidos en condiciones estrictas de laboratorio varían en relación a los obtenidos en un ambiente variable como lo es la iluminación en el interior de la comunidad vegetal, éste es el caso de $P$. aequale que en condiciones de laboratorio por lo general no germina en RL (Fig. 2) (Orozco-Segovia y Vázquez-Yanes, 1989).

Las condiciones de maduración de los frutos puede ser variable. Por ejemplo las infrutescencias de $P$. auritum maduran bajo valores de R/RL que van de 0.22 a 0.75 y las proporciones del fruto y de segmentos de éste sujetos a esta gama de valores varía dependiendo de la posición del fruto en la rama. Esto debe reflejarse en los contenidos de Pfr/Pt con los que son liberadas las semillas al alcanzar diferentes fotoequilibrios bajo las condiciones de luz de cada segmento (datos no publicados). En otras especies como $P$. hispidum se han encontrado variaciones en la respuesta a la luz de semillas procedentes de diferentes localidades (Ludlow y Vázquez-Yanes, 1976).

También condiciones de iluminación variable pueden inducir diferentes posibilidades de germinación sobre la superficie del suelo. En un experimento que duró dos meses las semillas de 4 especies de Piper fueron almacenadas imbibidas en cajas de Petri sobre la superficie del suelo y se observaron dos patrones de respuestas: $P$. aff hispidum y $P$. aequale germinaron prácticamente en su totalidad, en esta condición mientras que $P$. umbellatum y $P$. auritum germinaron en relación con el microambiente lumínico bajo el que quedaron, en algunas cajas hubieron altos porcentajes de germinación, pero en gran parte la germinación fue muy baja (Fig. 3). Estas respuestas probablemente no sólo son el reflejo de condiciones de iluminación variables, en el piso de la selva, sino también de la heterogeneidad en el contenido de Pfr/Pt inicial de las semillas y del umbral de respuesta de cada especie (Orozco-Segovia, 1986). 

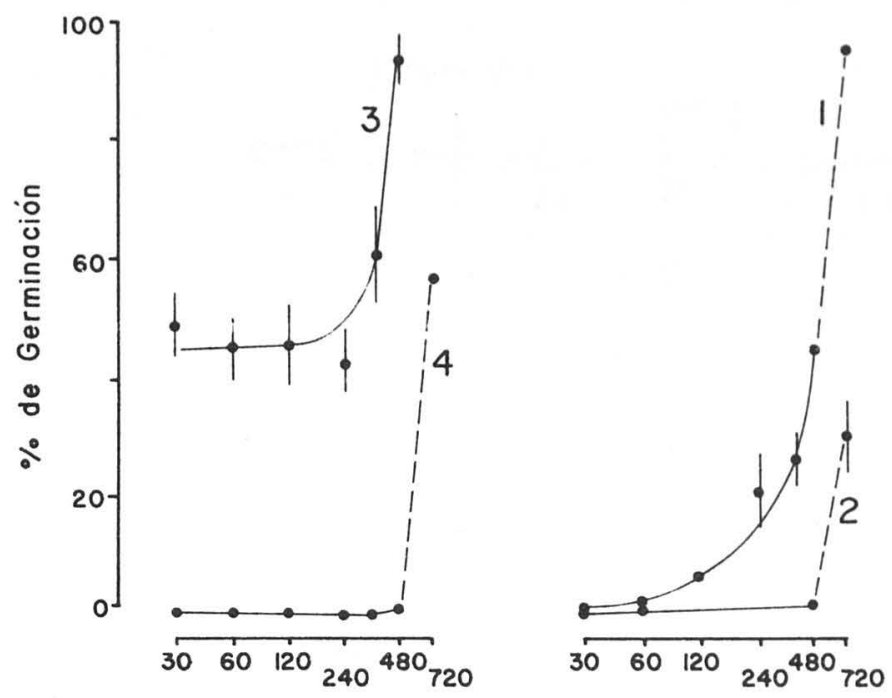

\section{Minutos $(\log )$}

Figura 1.- Respuesta a diferentes tiempos de exposición a la luz blanca y roja lejana en condiciones de laboratorio. Las semillas permanecieron en cajas de acrílico con luz rica en rojo lejano $12 \mathrm{~h}$, durante 13 días, este período fué interrumpido con luz blanca por los tiempos indicados en la escala logarítmica, el fotoperiodo diario fué completado con oscuridad. La línea punteada corresponde a la germinación obtenida en condiciones de sombra artificial $(12 \mathrm{~h})$.

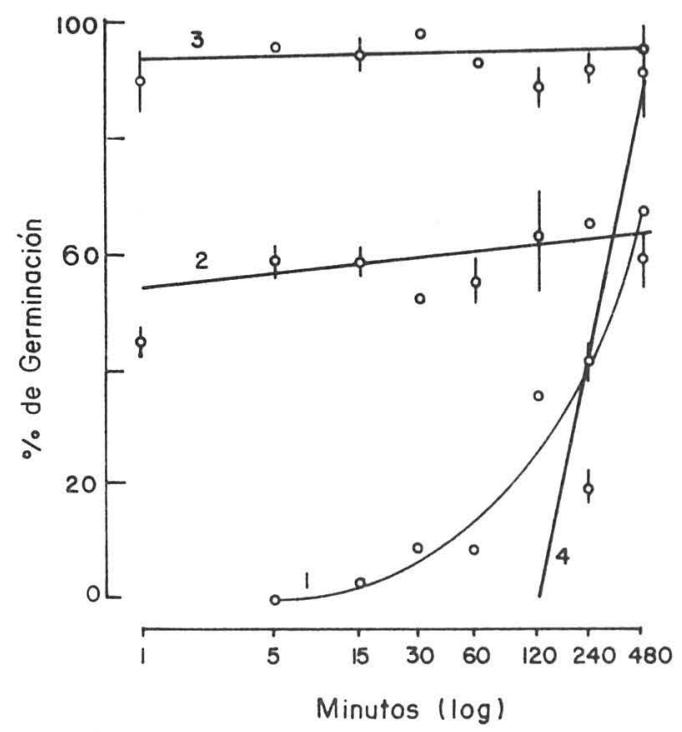

Figura 2.- Respuesta a diferentes tiempos de exposición a luz completa y filtrada por un dosel vegetal en condiciones de campo. Las semillas permanecieron en el piso de la selva, recibiendo luz rica en rojo lejano, $12 \mathrm{~h}$, durante 13 días; este período fué intcrrumpido con luz blanca por los tiempos indicados en la escala logarítmica, el fotoperiodo diario fué completado con oscuridad. 

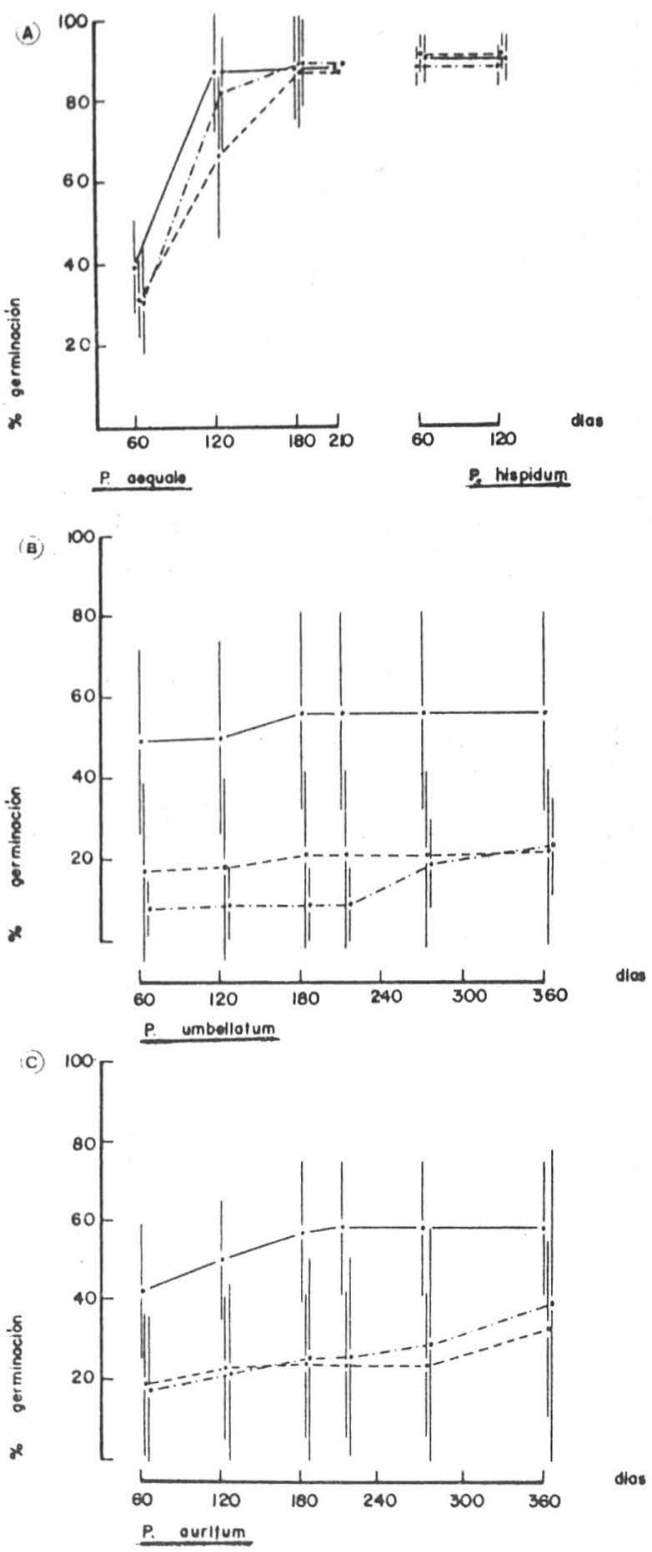

Figura 3.- Se muestra la germinación de semillas que estuvieron sembradas en cajas de Petri con agar bajo la copa de 3 árboles de la selva durante diferentes lapsos de tiempo árbol 1 (-), árbol 2 (---), árbol 3 (-.-). 
En experimentos hechos sobre el efecto del tiempo de almacenamiento en condiciones de laboratorio en semillas de los géneros Eupatorium, Myriocarpa, Piper y Urera se ha encontrado que las semillas almacenadas en seco conservan, por lo general, las características de su respuesta a la luz y en gran porcentaje su viabilidad (Tabla 1). En $U$. caracasana la modificación más importante, relacionada con su respuesta a la luz es la pérdida del requerimiento de alternancia de temperatura para alcanzar altos porcentajes de germinación.

En estas mismas especies en semillas almacenadas imbibidas en la oscuridad hay poca variación en su capacidad para germinar después de periodos de tiempo mayores de un año, sin embargo en un estudio realizado con 4 especies del género Piper se encontró que dos de ellas modificaron algunas de las características de su respuesta a la luz, $P$. umbellatum y $P$. aff hispidum, germinan después de algún tiempo de estar almacenadas en esta condición al exponerlas al RL, aún en condiciones de laboratorio (Tabla 1). Estos experimentos permiten suponer que las condiciones de humedad en las que quedan las semillas después de la diseminación son importantes para el comportamiento posterior de la semilla (Orozco-Segovia, 1986).

Las semillas enterradas en el suelo pueden sufrir fuertes modificaciones en su respuesta a la luzy en su germinabilidad, Por ejemplo $P$. auritum y $P$. aff hispidum pueden perder su capacidad para germinar a los 10 meses de enterramiento, y después de 2 meses de estar en esta condición pueden germinar en la oscuridad y en el RL al ser extraídas del suelo. Mientras que otras especies como $E$. galeotti, $M$. longipes, $P$. umbellatum, $P$. aequale y $U$. caracasana no germinan en la oscuridad al ser extraídas del suelo después de lapsos de tiempo tan largos como 16 meses (Tabla 2) por último señalaremos que algunas de las especies como $P$. umbellatum puede germinar al ser expuesta al RL después de 4 meses de enterramiento (Orozco-Segovia y VázquezYanes, 1989).

Sin embargo es importante señalar que experimentos realizados con semillas enterradas de $P$. auritum y $P$. hispidum, utilizando una metodología similar en todos los casos, pero en diferentes micrositios, con otros cohortes de semillas y en diferentes años sugieren que las condiciones del suelo no siempre tienen el mismo efecto sobre las mismas especies. Por lo menos en la capacidad para germinar al ser extraídas del suelo se encuentran grandes variaciones, ya que en algunos casos pueden conservar, en gran porcentaje, su capacidad para germinar por más de un año y en otros no (Vázquez-Yanes y Smith, 1982; Pérez-Nasser, 1985; Orozco-Segovia, 1986).

Por otra parte las diferentes vías por las cuales una semilla llega al suelo pueden afectar a las semillas de diferente manera, en experimentos realizados con C. obtusifolia se ha observado que después de ser ingeridas y excretadas por murciélagos (Artibeus jamaicensis) (AJ) las semillas no tienen diferencias significativas en su comportamiento a la luz ni en su capacidad para germinar en relación a semillas recién colectadas (RC), sin embargo cuando han sido ingeridas por monos araña (Ateles geofroyii) (AG) o cabezas de viejo (Eira barbara) (EB) que tienen un proceso digestivo más lento, se modifica la transmitancia de la cubierta de la semilla (se vuelven más opacas), se reduce considerablemente la viabilidad, hay destrucción de un gran número de semillas y se reduce el mecanismo de control del fitocromo pudiendo germinar aún en RL y parcialmente en la oscuridad. Sin duda alguna los efectos de la dispersión y de los factores del 
Tabla 1. Germinación de semillas recién colectadas, almacenadas durante un año, en seco en el laboratorio e imbibidas en la oscuridad. En las 5 últimas especies se verificó si las condiciones de almacenamiento tenían algún efecto en la respuesta de las semillas al rojo lejano y a la oscuridad, en ningún caso hubo germinación en la oscuridad. *especies que germinaron en rojo lejano.

\begin{tabular}{|c|c|c|c|}
\hline \multirow[b]{2}{*}{ Especie } & \multirow[b]{2}{*}{ Inicial } & \multicolumn{2}{|c|}{$\begin{array}{l}\text { \% de germinación } \\
\text { Almacenadas }\end{array}$} \\
\hline & & en seco & embibidas en la oscuridad \\
\hline Eupatorium galeotti & $33(3)$ & $20(13)$ & $27(15)$ \\
\hline Myriocarpa & $11(5)$ & $16(5)$ & $13(4)$ \\
\hline longipes & $48(2)$ & $-\cdots$ & $45(4)$ \\
\hline Piper aequale & $91(4)$ & $58(1)$ & 91 (1) \\
\hline P. auritum & $97(1)$ & $95(2)$ & $91(1)$ \\
\hline P. hispidum & $97(3)$ & $82(2)$ & $86(6)^{*}$ \\
\hline P. umbellatum & $95(3)$ & $81(2)$ & $86(3)^{*}$ \\
\hline Urera caracasana & $85(10)$ & $89(3)$ & $83(3)$ \\
\hline
\end{tabular}




\section{FITOCROMO}

Tabla 2. Germinación de semillas recién colectadas y después de estar un año enterradas en el suelo de la selva. *información obtenida de Pérez-Nasser, 1984. ( ) desviación stándard.

\section{\% de germinación}

Especie Inicial Después de un año de enterradas

Cecropia obtusiofolia

Eupatorium galeotti

Myriocarpa longipes

Piper aequale

P. auritum

P. umbellatum

Urera caracasana
84 (1)

$43(8)^{*}$

33 (3)

0

57 (6)

$19(4) *$

11 (5)

0

91 (4)

49 (10)

89 (2)

$16(6)^{*}$

96.6 (1)

4 (3)

98

76 (13)

68

$24(20)^{*}$

96.6 (3)

0

95 (3)

$36(8)$

85 (10)

70 
Tabla 3. Respuesta a la luz y a la oscuridad y fotorreversiones en semilias, provenientes de diferentes fuentes, de Cecropia obtusifolia. El subíndice 3 indica el número de horas que duró cada tratamiento por día. La germinación reportada para luz y oscuridad representa la germinación real en la muestra. El resto son datos extrapolados a un $100 \%$ de la viabilidad total de las semillas en la muestra. Los sím- bolos usados para las poblaciones de semillas se encuentran explicados en el texto.

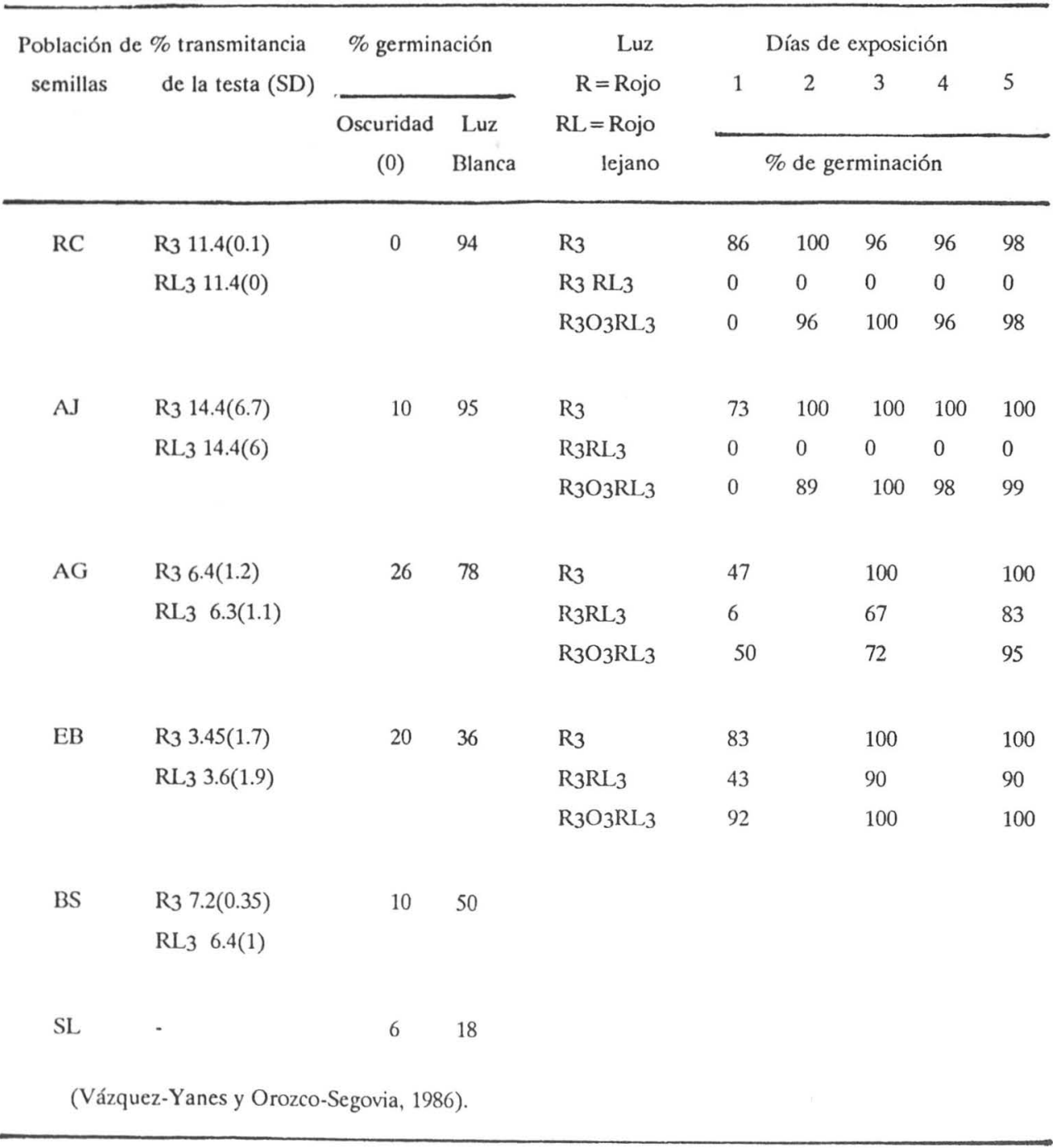


suelo se reflejan en la respuesta a la luz y en la capacidad para germinar de semillas extraídas directamente del suelo, en las que se ha observado germinación en la oscuridad y una reducida capacidad para germinar (SL); esto mismo se encontró en semillas enterradas dentro de bolsas de tela nylon en el suelo de Los Tuxtlas (BS) (Tabla 3) (Vázquez-Yanes y Orozco-Segovia, 1986).

Por último, cuando se han puesto a germinar muestras del suelo de la selva, en condiciones de luz controlada, se ha encontrado que hay una emergencia de plántulas significativamente mayor en tratamientos con LB y R en relación con la obtenida en RL y en la oscuridad, lo que sin duda alguna indica que la luz es el factor que regula la germinación de muchas de las especies presentes en el banco de propágulos del suelo (datos no publicados). En condiciones de campo Salmerón (1984) obtiene resultados similares a pesar de que en su trabajo hay factores que por razones obvias no pueden ser controladas como: humedad y temperatura.

\section{DisCUSIÓN}

El hecho es que las semillas fotoblásticas esten presentes en el suelo formando parte del banco de semillas, ya sea porque han conservado su mecanismo de latencia inicial o porque han adquirido otro a partir de éste, ya que las semillas que después de un tiempo de enterramiento son capaces de germinar en la oscuridad, sólo germinan al ser extraídas del suelo.

Falta mucho por conocer en relación al fotoblastismo y a su participación en la dinámica de las comunidades vegetales, ya que el banco de propágulos es una entidad dinámica que está formado por semillas que difieren entre sí en la época en que fueron producidas, en los progenitores, en el tiempo de permanencia en el suelo, eventos que los llevaron hasta él, microambientes ocupados, etc., y por lo tanto con diferentes capacidades de germinar y establecerse. A esto hay que sumarle el efecto de los predadores y las fluctuaciones de sus poblaciones en el espacio y en el tiempo.

Sin duda alguna la complejidad de los hechos que llevan a las semillas al suelo, a la germinación y a su establecimiento son los responsables de que la recolonización sea un evento variable; sin embargo, los refinados mecanismos de detección de la luz de las especies fotoblásticas deben permitir a éstas, por lo menos en periodos cercanos a su llegada al suelo detectar con precisión las condiciones ambientales, para después germinar o incorporarse al banco de semillas de acuerdo con las condiciones encontradas.

El fotoblastismo parece no tener siempre el mismo significado para todas las especies y esto no sólo depende de los requerimientos de germinación y de establecimiento de cada una de ellas, si no que la sensibilidad a la luz puede tener un valor diferente en el espacio y en el tiempo dentro de la comunidad vegetal.

Para aclarar este panorama falta conocer mucho sobre los cambios del fitocromo en diferentes condiciones de hidratación, sobre las variaciones que dependen de las condiciones de formación de la semilla, conocer si la pérdida de la capacidad de germinación es permanente, o temporal como ocurre en semillas del suelo en zonas templadas, en general mayor número de investigaciones sobre los aspectos aquí presen- 
tados, y de esta manera aclarar cuales son las posibilidades de participación en la dinámica de las comunidades de las semillas recién llegadas en relación con las que llevan más tiempo en el suelo, ya que esto va a depender de los eventos ocurridos desde el momento de formación de las semillas, e incluso de sucesos previos. 


\section{FITOCROMO}

\section{LITERATURA CITADA}

ALEXANDER, H. M. y R. D. WULFF. 1985. Experimental ecological genetics in Plantago, X: The effects of maternal temperature on seed and seedling characters in P. lanceolata. J. Ecology 73: 271-282.

BLISS, D. y H. SMITH 1985. Penetration of light into soil and its role in the control of seed germination. Plant Cell and Environment 8: 475-483.

BORTHWICK, H.A., S. B. HENDRICKS, M.W.T. PARKER y V. K. TOOLE. 1952. A reversible photoreaction controlling seed germination. Proc. Nat. Acad. Sci. 38: 662-666.

COME, D. 1970. Les obstacles a la germination. Masson. et cie editeurs, Paris. 162 p.

CRESSWELL, E. G. y J. P. GRIME. 1981. Induction of a light requirement during seed development and its ecological consequences. Nature 291: 583-585.

CUMMING, B. G. 1963. The dependence of germination photoperiod ligth quality and temperature in Chenopodium spp. Canad. J. Bot. 41: 1211-1233.

ESTRADA, A., R. COATES-ESTRADA y M. MARTINEZ-RAMOS. 1985. La estación de biología tropical "Los Tuxtlas" un recurso para el estudio y conservación de las selvas del trópico húmedo. pp. 379-393. En: Gomez-Pompa, A. y S. Del Amo. (Edrs.). Investigaciones sobre la regeneración de selvas altas en Veracruz, México, Vol. II. INIREB. Ed. Alhambra, México.

EVENARI, M. 1965. Light and seed dormancy. pp 804-847. En: W. Ruhland (Edr.). Handb. Planzenphysiol. 15 (2). Springer Verlag, Berlin.

FELTNER, K. C. y J. F. VASECKY. 1968. Light quality and temperature effects on weed seed germination in two Kansas soils. Trans. Kansas Acad. Sci. 71:7-12.

FENNER, M. 1985. Seed ecology. Chapman \& Hall L.T.D, London.

GUTTERMAN, Y. 1974. The influence of the photoperiodic regime and red far-red light treatments of Portulaca oleracea L. plants on the germinability of their seeds. Oecologia (Berlin) 17: 27-38.

KARSSEN, C. M. 1980/81a. Environmental conditions and endogenous mechanisms involved in secondary dormancy of seeds. Israel J. Bot. 29: 45-64.

KARSSEN, C. M. 1980/81b. Patterns of change in dormancy during burial of seeds in soil. Israel J. Bot. 29: $65-73$.

KIGEL, J., A. GIBLY. y M. NEGBI. 1979. Seed germination in Amaranthus retroflexus L. as affected by the photoperiod and age during flower induction of the parent plants. J. Exp. Bot. 30: 997-1002.

KIGEL, J., M. OFIR. y D. KOLLER. 1977. Control of the germination responses of Amaranthus retroflexus L. seeds by their parental phototermal environment. J. Exp. Bot. 28: 1125-1136.

KOLLER, D. 1962. Preconditioning of germination in lecttuce of time of fruit ripening. Am. J. of Botany 49: 841-844.

LUDLOW, B. y C. VAZQUEZ-YAÑES. 1976. Germinación de semillas de P. hispidum bajo diferentes condiciones de iluminación. pp. 263-278. En: Gómez-Pompa, A., C. Vázquez-Yanes, Del Amo, S. y A. Butanda (Edrs.). Regeneración de selvas. Ed. Continental, México.

MARTINEZ-RAMOS, M. y E. ALVAREZ-BUYLLA. 1986. Seed dispersal, gap dynamics and tree recruiment: The case of Cecropia obtusifolia at Los Tuxtlas, Mexico. pp. 333-346. En: Estrada, A. y T. H. Fleming (Edrs.). Frugivores and seed dispersal. T:VS 15. Dr W. Junk Publishers. The Hague, Netherlands. pp 333-346. 
MIRANDA, F. y E. HERNANDEZ X. 1963. Los tipos de vegetación de México y su clasificación. Bol. Soc. Bot. México 28: 27-178.

OROZCO-SEGOVIA, A. 1986. Fisiología ecológica del fotoblastismo en semillas de cuatro especies del género Piper L. Tesis de Doctorado UNAM, México.

OROZCO-SEGOVIA, A., C. VAZQUEZ-YANES, R. COATES-ESTRADA y N. PÉREZ-NASSER. 1987. Ecophysiological characteristics of the seed of the tropical forest pioneer Urera caracasana (Urticaceae) Tree physiology 3:375-386.

OROZCO-SEGOVIA, A. y C. VAZQUEZ-YANES. 1989. Light effect on seed germination in Piper L. Acta Oecológica (Oecol. Plant.) 10:123-146.

PÉREZ-NASSER, N. 1985. Viabilidad en el suelo de las semillas de once especies de la vegetación de Los Tuxtlas, Ver. Tesis Fac. Ciencias, UNAM, México.

SALMERON, R. 1984. Germinación de semillas acumuladas en el suelo de una selva húmeda tropical "Los Tuxtlas", Veracruz. México. Tesis Fac. Ciencias, UNAM, México.

SATTER, R. L. y A. W. GALSTON. 1977. The physiological functions of phytochrome. pp. 680-734. En: T. W. Goodwin (Edr.). Chemistry and Photochemistry of plant pigments. Academic Press, London.

SMITH, H. 1982. Light quality photoperception and plant strategy. Ann. Rev. Plant. Physiol. 33: 481-518. VAZQUEZ-YANES, C. 1976. Estudios sobre ecofisiología de la germinación en una zona calido-humeda de México. pp. 279-387. En: Gómez-Pompa, A., C. Vázquez-Yanes, S. Del Amo y A. Butanda (Edrs.). Regeneración de selvas. Editorial Continental, México.

VAZQUEZ-YANES, C. y A. OROZCO-SEGOVIA. 1982. Germination of the seeds of a tropical rainforest shrub, Piper hispidum Sw. (Piperaceae) under different light qualities. Phyton 42:143-149.

VAZQUEZ-YANES, C. y A. OROZCO-SEGOVIA. 1984. Ecophysiology of seed germination in the tropical humid forest of the world: A review. pp. 37-50. En: Medina, E., H. A. Mooney and C. Vázquez-Yanes (Edrs.). Physiological Ecology of plants of the wet tropics. T:VS 12. Dr. W. Junk Publisher. The Hague Netherlands.

VAZQUEZ-YANES, C. y A. OROZCO-SEGOVIA. 1986. Dispersal of seeds by animals: effect on light controlled dormancy in Cecropia obtusifolia. pp 333-346. In: Estrada, A. y T. H. Fleming (Edrs.). Frugivores and seed dispersal. T:VS 15. Dr. W. Junk Publishers. The Hague, Netherlands.

VAZQUEZ-YANES, C. y A. OROZCO-SEGOVIA. 1987. Light gap detection by the photoblastic seeds of Cecropia obtusifolia and Piper auritum, two tropical rain forest trees. Biol. Plant. (Praha). 29(3): 234-236.

VAZQUEZ-YAÑES, C. y H. SMITH. 1982. Phytochrome control of seed germination in the tropical rain forest pioneer trees (Cecropia obtusifolia y Piper auritum) and its ecological significance, New Phytol. 92: $477-485$.

WESSON, G. y P. F. WAREING. 1969a. The induction of light sensivity in weed seeds by burial J. Exp. Bot. 20: 214-225.

WESSON, G. y P. F. WAREING. 1969b. The role of light in the germnation of naturally occurring populations of buried seeds. J. Exp. Bot. 20: 402-413.

WHITMORE, T. C. 1983. Secondary succession from seed in tropical rain forest. Forestry Abstracts 44: 767-779.

WURZBURGER, J. y D. KOLLER. 1976. Differential effects of the parental phototermal environment on development of dormancy in caryopses of Aegilops kotschyi. J. Exp. Bot. 27: 43-48. 